ARTICLE

https://doi.org/10.1038/s41467-020-19526-x

\title{
Free-space optical delay line using space-time wave packets
}

Murat Yessenov (1D ${ }^{1}$, Basanta Bhaduri ${ }^{1}$, Peter J. Delfyett ${ }^{1} \&$ Ayman F. Abouraddy (D) ${ }^{1 凶}$

An optical buffer featuring a large delay-bandwidth-product-a critical component for future all-optical communications networks-remains elusive. Central to its realization is a controllable inline optical delay line, previously accomplished via engineered dispersion in optical materials or photonic structures constrained by a low delay-bandwidth product. Here we show that space-time wave packets whose group velocity is continuously tunable in free space provide a versatile platform for constructing inline optical delay lines. By spatiotemporal spectral-phase-modulation, wave packets in the same or in different spectral windows that initially overlap in space and time subsequently separate by multiple pulse widths upon free propagation by virtue of their different group velocities. Delay-bandwidth products of $\sim 100$ for pulses of width $\sim 1$ ps are observed, with no fundamental limit on the system bandwidth.

${ }^{1}$ CREOL, The College of Optics and Photonics, University of Central Florida, Orlando, FL 32816, USA. ${ }^{\varpi_{e m a i l}}$ raddy@creol.ucf.edu 
T he relentless increase in demand for communications bandwidth ${ }^{1}$ has spurred developments in sub-systems that are critical for future optical networks, such as spatialmode multiplexing ${ }^{2-4}$ and ultrafast modulators ${ }^{5}$. A critical-but to date elusive-component is an optical buffer that alleviates data packet contention at optical switches by reordering the packets without an optical-to-electronic conversion ${ }^{6}$. Previous efforts addressing this challenge have exploited so-called slow light ${ }^{7,8}$, which refers to the reduction in the group velocity of a pulse traversing a selected material ${ }^{9-12}$ or carefully designed photonic structure ${ }^{13-15}$. Despite the diversity of their physical embodiments ${ }^{16}$, slow-light approaches typically rely on resonant optical effects and are thus limited by a delay-bandwidth product (DBP) on the order of unity. That is, the differential group delay with respect to a reference pulse traveling at $c$ (the speed of light in vacuum) does not exceed the pulse width ${ }^{17,18}$, which falls short of the requirements of an optical buffer ${ }^{19}$. Time-varying systems ${ }^{20}$ can overcome this limit at the expense of increased implementation complexity. In one realization, a trapdoor mechanism in coupled resonators increases the storage time through externally controlled coupling ${ }^{21}$-but losses concomitantly increase in step with the delay. An alternate approach makes use of non-resonant recycling of orthogonal modes in a cavity ${ }^{22}$. A different strategy relies on transverse spatial structuring to reduce the group velocity in free space, but only a minute reduction below $c$ has been detected to date ${ }^{23,24}$. Nevertheless, theoretical proposals suggest that pushing this approach to the limit may produce sufficiently large differential group delays for an optical buffer ${ }^{25,26}$, but dispersive spreading is associated with the propagation of these wave packets ${ }^{27}$. Finally, a recent theoretical proposal suggests that optical non-reciprocity can help bypass the usual DBP limits ${ }^{28}$, but doubts have been cast on this prospect ${ }^{29,30}$.

An altogether different approach for tuning the group velocity of a pulse makes use of space-time (ST) wave packets: propagation-invariant pulsed beams (diffraction-free and dispersion-free) $)^{31-38}$ endowed with structured spatio-temporal spectra $^{39-43}$ in which each spatial frequency is associated with a single wavelength ${ }^{44-49}$. Although it has long been known theoretically that ST wave packets may take on arbitrary group velocities (speed of the wave-packet peak) in free space ${ }^{49-53}$, experiments have revealed group-velocity deviations from $c$ of only $\sim 0.1 \%^{54-56}$, corresponding to group delays of tens or hundreds of femtoseconds-several orders-of-magnitude below the requirements for an optical buffer. We recently introduced a spatio-temporal phase-only synthesis strategy for the preparation of ST wave packets that allows fully exploiting their unique characteristics ${ }^{57}$. For example, we have reported free-space group velocities tunable from $30 c$ to $-4 c^{58}$, group delays of $\sim 150 \mathrm{ps}^{59}$, propagation distances extending to $70 \mathrm{~m}^{60,61}$, among other unique properties ${ }^{62-65}$. These attributes indicate the potential utility of ST wave packets in constructing an optical buffer.

Here we report a proof-of-principle realization of a readily controllable, inline, free-space optical delay line-the centerpiece of an optical buffer-utilizing the tunable group velocity of ST wave packets. By assigning to optical pulses different group velocities (whether subluminal, superluminal, or negative), the resulting wave packets separate from each other upon free propagation by multiple pulse widths. We observe a differential group delay of $\sim 160$ ps between two wave packets of widths as small as $\sim 1.3 \mathrm{ps}$, corresponding to a DBP of $\sim 100$, which is $\sim 2$ orders-of-magnitude larger than previous demonstrations ${ }^{19}$. We realize several desiderata for an all-optical buffer, such as introducing a controllable delay between two pulses (or more) in the same or different spectral channels, while occupying the same or different spatial modes. There is no need here for the widths of the spectral channels to be equal nor do they need to be mutually coherent. It is critical to note that tuning the group velocity is not associated with changes in the pulse amplitude. Furthermore, subluminal and superluminal wave packets are treated on the same footing, in contrast to the typically distinct approaches of slow-light and fast-light, which highlights the versatility of our strategy. Crucially, the group delay introduced between the wave packets in free space is independent of the external degrees of freedom of the field (such as wavelength). Moreover, due to the absence of limitations stemming from resonant effects, there are in principle no bandwidth constraints. Although the spectral resolution of the system imposes a limit on the maximal differential group delay ${ }^{59}$, there are no limits on the pulse width that can be exploited. Consequently, future increases in transmission rates can be accommodated without changing the system architecture.

\section{Results}

A free-space optical delay line based on ST wave packets. Our overall conceptual scheme is illustrated in Fig. 1a. Optical pulses that overlap in time and travel in free space at a group velocity $\widetilde{v}=c$ are transformed into ST wave packets having different group velocities $\widetilde{v}_{1}$ and $\widetilde{v}_{2}$ via spatio-temporal spectral phase modulations implemented independently in each spectral channel. By virtue of their different group velocities, the wave packets gradually separate in time by a group delay that depends on the difference between their group velocities and the propagation distance.

The impact of the phase modulation can be appreciated by examining the spectral space $\left(k_{x}, k_{z}, \frac{\omega}{c}\right)$, where $k_{x}$ and $k_{z}$ are the transverse and axial components of the wave vector, and $\omega$ is the angular frequency, which satisfy the free-space dispersion relationship $k_{x}^{2}+k_{z}^{2}=\left(\frac{\omega}{c}\right)^{2}$ that is represented geometrically as the light-cone. We assume the field is uniform along the other transverse coordinate $y\left(k_{y}=0\right)$, and refer to $k_{x}$ and $\omega$ as the spatial and temporal frequencies, respectively. The spatiotemporal spectra of the initial plane-wave pulses lie along the tangent to the light-cone at $k_{x}=0$. The spectral phase modulation is designed to rotate the tangential plane by a spectral tilt angle $\theta$ with respect to the $k_{z}$-axis. The result is a propagation-invariant wave packet traveling at a group velocity of $\widetilde{v}=c \tan \theta$, where $\widetilde{v}$ is the velocity of the central peak of the wave packet. Although $\widetilde{v}$ can take on arbitrary values, this does not entail a violation of relativistic causality $59,66-69$, as we explain further below. We delineate three regimes ${ }^{70}: 0<\theta<45^{\circ}$ is subluminal $\widetilde{v} ; 45^{\circ}<\theta<90^{\circ}$ is superluminal $\widetilde{v}$; and $90^{\circ}<\theta<$ $180^{\circ}$ is a negative- $\widetilde{v}$ domain $\widetilde{v}<0$. In the subluminal regime, the spatio-temporal spectral locus of the wave packet is an ellipse, and is a hyperbola, parabola, or ellipse in the superluminal and negative- $-\tilde{v}$ regimes depending on the value of $\theta$ (Fig. 1a, insets); see "Methods" section.

Previous experiments ${ }^{34,71,72}$, including our group's ${ }^{42,58,59,64}$, have all been restricted to synthesizing single ST wave packets of a given group velocity. Here, we synthesize up to three ST wave packets inline simultaneously-each wave packet having a different group velocity - by implementing a segmented phase modulation scheme that addresses each spectral channel separately (Fig. 1a, inset). Therefore, the initially overlapping wave packets travel rigidly in free space at their respective group velocities without diffraction or dispersion, thereby separating from each other (see "Methods" section). A large number of wave packets can be addressed in this fashion using large-area phase plates for spectral-phase modulation ${ }^{73}$, and the widths of the spectral channels need not be equal nor the spectra have mutual coherence ${ }^{74,75}$. 

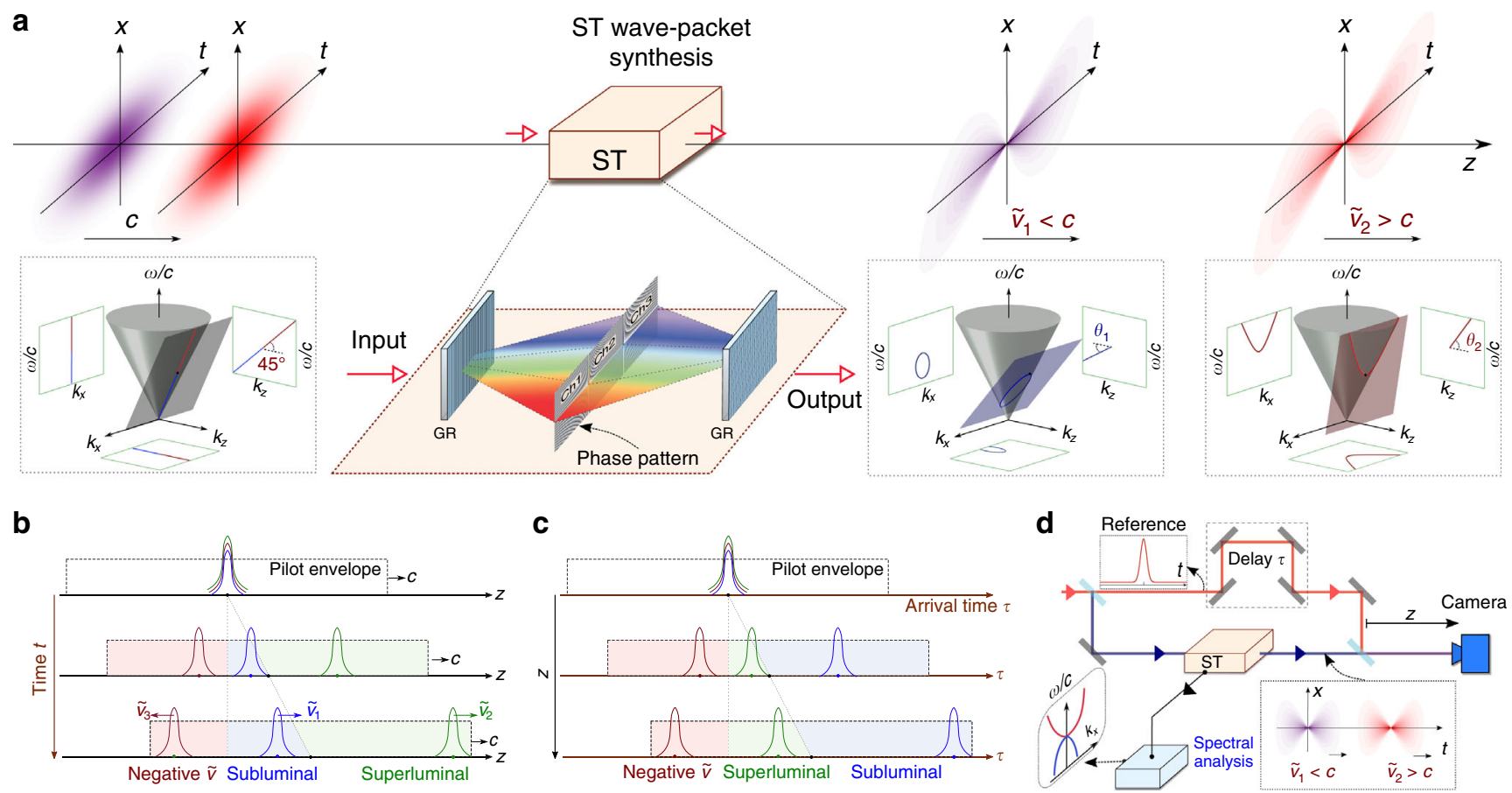

Fig. 1 Concept of a free-space optical delay line using ST wave packets. a Overall layout. Traditional pulses whose group velocities are equal $(\widetilde{v}=c)$ are converted into ST wave packets each assigned a different group velocity (subluminal $\widetilde{v}_{1}$ and superluminal $\widetilde{v}_{2}$ ), so that they separate in time upon free propagation. The spatio-temporal spectrum of the traditional pulses and the ST wave packets are depicted on the surface of the light-cone $k_{x}^{2}+k_{z}^{2}=\left(\frac{\omega}{c}\right)^{2}$, and their spectral projections are also shown (straight line for a plane-wave pulse, an ellipse for a subluminal ST wave packet, and a hyperbola for a superluminal ST wave packet). Here $\widetilde{v}=c \tan \theta$, where $\theta$ is the spectral tilt angle with respect to the $k_{z}$-axis. Inset shows the structure of the ST wave packet synthesizer for multiple spectral channels (Ch1, Ch2, and Ch3); GR: Diffraction grating. b Dynamics of ST wave packets under the pilot envelope. The locations of subluminal, superluminal, and negative- $\widetilde{v}$ ST wave packets are shown along the propagation axis $z$ at different instances in time. c The arrival times $\tau$ of ST wave packets are shown at different detector locations along z. d Schematic of the optical setup for synthesizing and characterizing ST wave packets.

The propagation dynamics of ST wave packets having different group velocities is depicted in Fig. 1b, c. The wave packets overlap at $z=0$, but no longer overlap later on: the superluminal wave packet has moved the farthest ahead in space (beyond the distance traveled by a luminal pulse); the subluminal wave packet lags behind; while a negative- $\widetilde{v}$ wave packet is located at a negative- $z$ position (Fig. $1 \mathrm{~b}$ ). In our experiments, rather than detecting the ST wave packets at different $z$ for fixed delay, we instead scan the arrival time $\tau$ at a fixed detector position (Fig. 1c). By moving the detector to a positive- $z$ location, the superluminal wave packet arrives earlier than a luminal pulse while the subluminal wave packet arrives later. The negative $-\tilde{v}$ wave packet arrives earlier at $z>0$ than at $z=0$, thereby indicating its backward propagation-as confirmed unambiguously in our measurements.

Experimental arrangement. Our experiments (setup shown in Fig. 1d) are carried out using plane-wave femtosecond pulses at a wavelength of $\sim 800 \mathrm{~nm}$ from a Ti:sapphire laser (Tsunami, Spectra Physics). Spatio-temporal spectral modulation is performed with a diffraction grating (Newport 10HG1200-800-1) and a spatial light modulator (SLM; Hamamatsu X10468-02) 42,57,58,64. A bandwidth of $\sim 1.5 \mathrm{~nm}$ is spread over the width of the SLM active area, which is subdivided into three spectral channels (Ch1, $\mathrm{Ch} 2$, and Ch3) each of width $\sim 0.5 \mathrm{~nm}$ and corresponding to pulses of width $\sim 4 \mathrm{ps}$. The measured spectra of the three channels are plotted in Fig. 2a. In contrast to the one-dimensional (1D) phase distributions exploited in traditional ultrafast pulse modulation $^{76,77}$, here a two-dimensional (2D) phase distribution jointly manipulates the spatial and temporal spectra. Within each spectral channel the $2 \mathrm{D}$ phase distribution produces a ST wave packet having a spectral tilt angle of $\theta$, as shown in Fig. 2b. We assign Ch1 the spectral tilt angle $\theta_{1}=30^{\circ}$, corresponding to the subluminal group velocity $\widetilde{v}_{1}=0.58 c$; $\mathrm{Ch} 2$ is superluminal with $\theta_{2}=70^{\circ}$ and $\widetilde{v}_{2}=2.75 c$; whereas $\mathrm{Ch} 3$ is negative $-\widetilde{v}$ with $\theta_{3}=120^{\circ}$ and $\widetilde{v}=-1.73 c$. Any other values could be implemented in these channels by simply adjusting the phase distribution, and any channel can be deactivated by setting the SLM phase distribution to zero. A spatial filter in the path of the wave packets eliminates any deactivated channel.

The spatio-temporal spectral intensity of the ST wave packet is recorded in $\left(k_{x}, \lambda\right)$ space by implementing a spatial Fourier transform on the spectrally resolved pulses ${ }^{42}$. We plot in Fig. $2 c$ the spatio-temporal spectra of the three channels captured simultaneously. The curvature of each spectrum is determined by the associated spectral tilt angle $\theta^{72}$. From this spectral measurement we obtain the spectral projection onto the $\left(k_{z}, \frac{\omega}{c}\right)$ space; see Fig. $2 \mathrm{~d}$. The slopes of the linear spectral projections onto the $\left(k_{z}, \frac{\omega}{c}\right)$ space provide independent confirmation of the group velocities $\left(\widetilde{v}=\frac{\partial \omega}{\partial k_{z}}\right)$. Finally, by recording the time-averaged intensity distribution of the ST wave packet upon propagation $I$ $(x, z)=\int d t|E(x, z, t)|^{2}$ with a CCD camera (The ImagingSource, DMK 33UX178), we confirm the diffraction-free propagation as shown in Fig. 2e for Ch2 (Ch1 and $\mathrm{Ch} 3$ are deactivated). We define $L_{\max }$ to be the distance at which $I\left(0, L_{\max }\right)=\frac{1}{e} I(0,0)$.

To characterize the spatio-temporal intensity profile of ST wave packets and measure their group velocity, we place the spatio-temporal synthesis arrangement in one arm of a Mach-Zehnder interferometer ${ }^{58,64}$ and place a temporal delay 
a
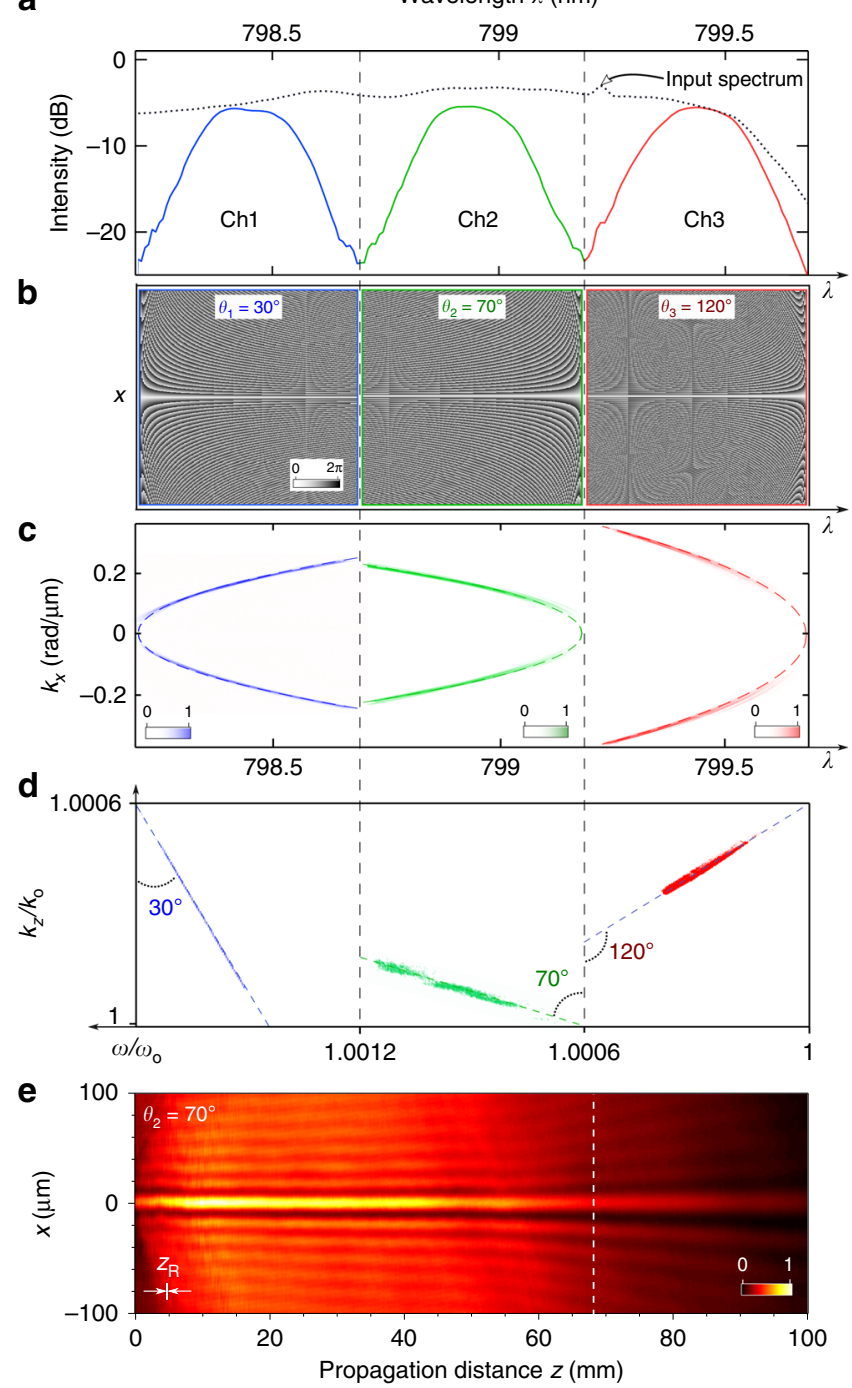

Fig. 2 Assigning different group velocities to ST wave packets in distinct spectral channels. a Spectra of the three spectral channels (solid curves) alongside the spectrum of the Ti:sapphire laser (dotted curve). $\mathbf{b}$ The 2D SLM phase distributions across the three channels to synthesize different ST wave packets: subluminal, Ch1; superluminal, Ch2; and negative- $\widetilde{v}$, Ch3. c Measured spatio-temporal spectrum $\left|\widetilde{E}\left(k_{x}, \lambda\right)\right|^{2}$ of the co-synthesized ST wave packets in the three spectral channels. Dashed curves are the theoretical expectations. d Spatio-temporal spectral projections onto the $\left(k_{z}, \frac{\omega}{c}\right)$-plane. The spectra for the different channels are shifted along the $k_{z}-$ axis to fall within the same range for clarity. e Measured time-averaged axial evolution of the intensity $I(x, z)=\int d t|E(x, z, t)|^{2}$ of $C h 2$. Note the difference in scale along $x$ and $z$. The thin white bar in the bottom-left corner corresponds to the Rayleigh range $z_{R}$ of a Gaussian beam having the same width as the ST wave packet. The vertical dashed line identifies $z=L_{\max }$.

in the other arm in the path of a reference pulse (100-fs pulses from the Ti:sapphire laser); see Fig. 1d. Such a measurement configuration corresponds to the conceptual scheme depicted in Fig. 1c. This technique yields only the intensity of the wave packet profile and not its phase, which requires more sophisticated spatio-temporal characterization tools ${ }^{55,78,79}$. Nevertheless, our approach suffices for estimating the arrival times and thence the group velocities ${ }^{58,64}$.

As seen in Fig. 2c (and also in Fig. 2d), the ideal correlation function between $k_{x}$ and $\omega$ is relaxed, mainly due to the finite spectral resolution of the diffraction gratings used. Ideal ST wave packets propagate indefinitely at an arbitrary group velocity but require infinite energy ${ }^{80}$, whereas realistic finite-energy ST wave packets maintain such group velocities over a bounded distance. Finite-energy ST wave packets are characterized by a spectral uncertainty $\delta \omega$ ( $\delta \lambda$ in wavelength), which corresponds to an unavoidable fuzziness in the association between spatial and temporal frequencies. In our experiments, we estimate a spectral uncertainty $\delta \lambda \sim 25 \mathrm{pm}^{59}$. Within this conception, a finite-energy ST wave packet can be decomposed into a product of an ideal ST wave packet traveling indefinitely at $\widetilde{v}$ (with a pulse width at the beam center proportional to the inverse of the bandwidth $\Delta \omega$ ) and a broad 'pilot envelope' propagating at a group velocity of $c$ (and whose width in time is the inverse of the spectral uncertainty $\delta \omega)$. Temporal walk-off between the ideal ST wave packet and the pilot envelope limits the propagation distance $L_{\max }$ of the ST wave packet and hence also constrains the maximum differential group delay $\tau_{\max }$ achieved with respect to a reference pulse traveling at $c$. It can be shown that $\tau_{\max } \sim \frac{1}{\delta \omega}$ and $L_{\max } \sim \frac{\mathcal{c \tau}_{\max }}{|1-\cot \theta|} 59$. Therefore, two ST wave packets having the same spectral uncertainty will travel different distances $L_{\max }$ according to their respective spectral tilt angles.

The pilot envelope traveling at $c$ ensures that no violation of relativistic causality occurs even for ST wave packets propagating at superluminal or negative $\widetilde{v}$. In such cases, when the wave packets reach the edge of the pilot envelope, they are suppressed and their spatio-temporal profile deformed ${ }^{59}$. This is associated with the drop in on-axis intensity values as shown in Fig. 2e. Figure 1b, c illustrate the propagation dynamics of three ST wave packets along the pilot envelope. The width of the pilot envelope represents an inherent temporal latency in our system. All ST wave packets emerge from the synthesis process after being delayed by some fixed amount (the temporal width of the pilot envelope, or the inverse of the spectral uncertainty) with respect to the input pulse. A superluminal ST wave packet can subsequently 'catch up' with the edge of the pilot envelope, but cannot exceed it ${ }^{59}$. Therefore, the information transmission speed does not exceed $c$.

Measurement results. We start by discussing the results of monitoring the propagation of a pair of ST wave packets of different group velocities (subluminal $\mathrm{Ch} 1$ and superluminal $\mathrm{Ch} 2$ ) in free space using our interferometric approach, which are plotted in Fig. 3. The spatio-temporal intensity profile is traced by scanning a 200-ps delay line in the reference arm of the MachZehnder interferometer (Fig. 1d) with the detector placed at different locations along $z$. The delay $\tau$ in the path of the reference pulse is adjusted first so that the reference pulse overlaps with the two ST wave packets when the detector is placed at $z=0$. At this point, the two ST wave packets overlap in space and time. The plane $z=0$ is arbitrary, and is set for convenience by arranging for appropriate delays in the paths of the ST wave packet and the reference pulse (Fig. 1d), which we select to provide the maximum distance for the ST wave packet to propagate ${ }^{59}$. At this plane, high-visibility spatially resolved interference fringes is evidence that the ST wave packet and the reference pulse overlap in space and time. Note the X-shaped spatio-temporal profile that is characteristic of most ST wave packets ${ }^{34}$. After moving the detector to $z=10 \mathrm{~mm}$, the wave packets are found to arrive at different times, which is verified by scanning the reference delay $\tau$. The superluminal pulse (on the left) arrives early and the subluminal pulse (on the right) arrives late with respect to a pulse traveling at $c$ whose position is indicated by a white circle in Fig. 3a. The delay between the two pulse centers arriving at axial position $z$ is $\Delta \tau=\frac{z}{\tilde{v}_{1}}-\frac{z}{\tilde{v}_{2}}=\frac{z}{c}\left\{\cot \theta_{1}-\cot \theta_{2}\right\}$. Therefore we 
a<smiles>C=CC(C)C</smiles>

Arrival time $\tau$

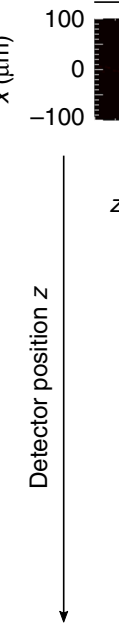

$-40$

0

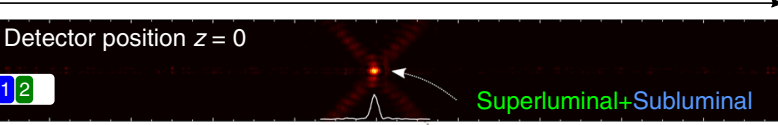

80 b

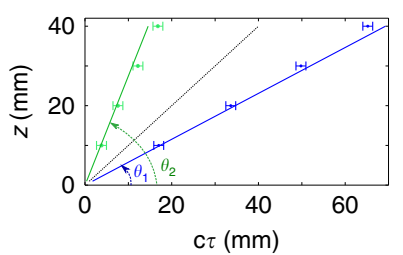

40

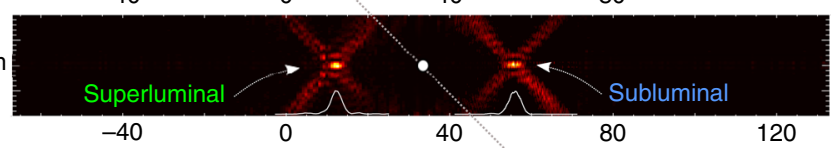

20
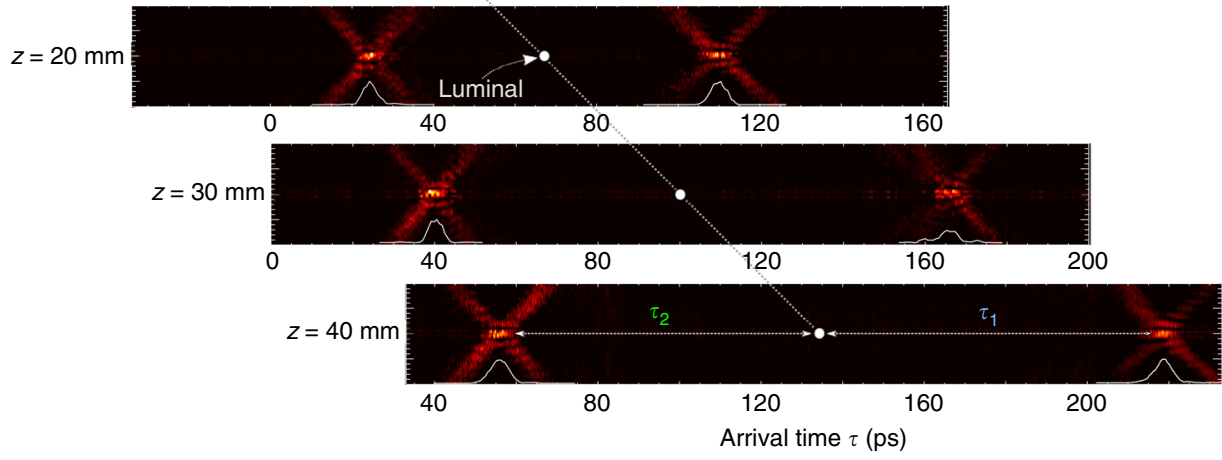

Fig. 3 Propagation of two ST wave packets having different group velocities in free space. a The panels display the arrival time of two wave packets (subluminal $\operatorname{Ch} 1$ with $\theta_{1}=30^{\circ}$ and superluminal $\operatorname{Ch} 2$ with $\theta_{2}=70^{\circ}$ ) at different detector planes $z$. When the detector is placed at $z=0$, the two wave

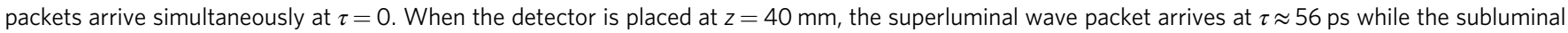
wave packet arrives at $\tau \approx 218 \mathrm{ps}$. In each panel we also plot the pulse profile at the wave packet center $x=0, I(0, z, \tau)$. The vertical and horizontal scales are adjusted so that the slope of the line traced by the center of each wave packet is $\tan \theta$, where $\theta$ is the spectral tilt angle. $\mathbf{b}$ Dynamics of the centers of the two wave packets revealing group velocities of $\widetilde{v}_{1}=c \tan \theta_{1}$ and $\widetilde{v}_{2}=c \tan \theta_{2}$. The points are experimental data and the straight lines are theoretical fits. The intermediate tilted line corresponds to a luminal pulse with $\widetilde{v}=c$.

expect that $\Delta \tau=45.6 \mathrm{ps}$ when the detector is placed at $z=10 \mathrm{~mm}$ and $\Delta \tau=182 \mathrm{ps}$ when $z=40 \mathrm{~mm}$; the corresponding measured values are $\Delta \tau \approx 44 \mathrm{ps}$ and $\Delta \tau \approx 162 \mathrm{ps}$, respectively. We note that the group delays far exceed the temporal widths $\Delta \tau$ of either wave packet at the center $x=0$, which corresponds to an effective delay-bandwidth product DBP $\gg 1$. The dynamics of the pulse centers are plotted in Fig. $3 \mathrm{~b}$ showing clearly the difference in their group velocities with respect to $c$. This is the first observation of two co-propagating optical wave packets separating from each other in free space without the influence of an optical material or photonic structure. Note that the spectral uncertainties for both ST wave packets are equal because their values are set by the grating size.

We next examine the dynamics of a different configuration where Ch1 and Ch3 are activated while Ch2 is deactivated, corresponding to subluminal and negative- $\widetilde{v}$ wave packets. After overlapping the ST wave packet and the reference pulse at $z=0$, we next move the detector to the plane $z=10 \mathrm{~mm}$ (note that we make use of only positive displacements of the detector along $z$ ). The interference fringes observed at $z=0$ disappear at $z=10 \mathrm{~mm}$ due to the relative group delay introduced between the ST wave packet (traveling at $\widetilde{v}$ ) and the reference pulse (traveling at $c$ ). The interference is subsequently regained by introducing the appropriate delay in the path of the reference pulse. As seen in Fig. 4a, the subluminal wave packet propagates in the forward direction while the negative- $\widetilde{v}$ wave packet travels backwards and arrives at the detector plane $z>0$ at $\tau<0$. In the case of negative- $\widetilde{v}$, the delay that needs to be introduced in order to regain the overlap between the two wave packets is negative; that is, less delay is to be inserted in this case with respect to the delay utilized when the detector was placed at $z=0$. In other words, the ST wave packet arrives at $z=$ $10 \mathrm{~mm}$ before its arrival at $z=0$. Finally, we activate Ch1, Ch2, and Ch3 simultaneously and observe their evolution, which is plotted in Fig. 4b. The dynamics of the wave packet centers as plotted in the insets of Fig. $4 \mathrm{a}$ and $\mathrm{b}$ confirm that the targeted group velocities have been realized. Once again, the spectral uncertainty is the same for all three channels.

After investigating the dynamics of ST wave packets sharing the same spatial channel but different spectral channels, we move on to the case of ST wave packets in the same spectral channel. First, two ST wave packets sharing Ch1 are assigned different group velocities $\left(\widetilde{v}_{1}\right.$ and $\left.\widetilde{v}_{2}\right)$ by interleaving the spectral phase patterns corresponding to the targeted spectral tilt angles $\left(\theta_{1}\right.$ and $\left.\theta_{2}\right)$; see Fig. $4 c$, inset. The measured spatio-temporal spectrum in $\left(k_{x}, \lambda\right)$ space reveals two overlapping spectra corresponding to subluminal and superluminal wave packets (compare to the displaced spectra for $\mathrm{Ch} 1$ and $\mathrm{Ch} 2$ in Fig. 2c). With free propagation, the two wave packets separate just as in the cases of wave packets in different spectral channels (Figs. 3 and Fig. 4a, b). Furthermore, the wave packets can be located in different spatial channels (along the transverse $x$-coordinate) by structuring the SLM spectral phase distribution along the direction that encodes the spatial frequencies $k_{x}$. An example is shown in Fig. $4 \mathrm{~d}$ where two ST wave packets are synthesized with group velocities $\widetilde{v}_{1}$ and $\widetilde{v}_{2}$ but with SLM phase distributions that are displaced vertically (rather than interleaved as in Fig. 4c), resulting in two wave packets that are in turn displaced along the transverse $x$-axis by $\sim 1.4 \mathrm{~mm}$.

A unique aspect of our strategy-in comparison with slow-light and fast-light approaches-is the absence of fundamental bandwidth constraints. Because we do not exploit an optical resonance, the bandwidth is in principle limited only by the experimental resources available. Increasing the utilized bandwidth (reducing the pulse width) does not affect the maximum differential group delay that is determined primarily by the spectral uncertainty ${ }^{59}$. To verify this, we confirm that the same group delay is obtained for a ST wave packet of fixed $\widetilde{v}$ as the pulse width is reduced from $\sim 4$ ps to $\sim 1 \mathrm{ps}$, corresponding to an increase in the DBP by a factor of $\sim 4$. Here the phase 

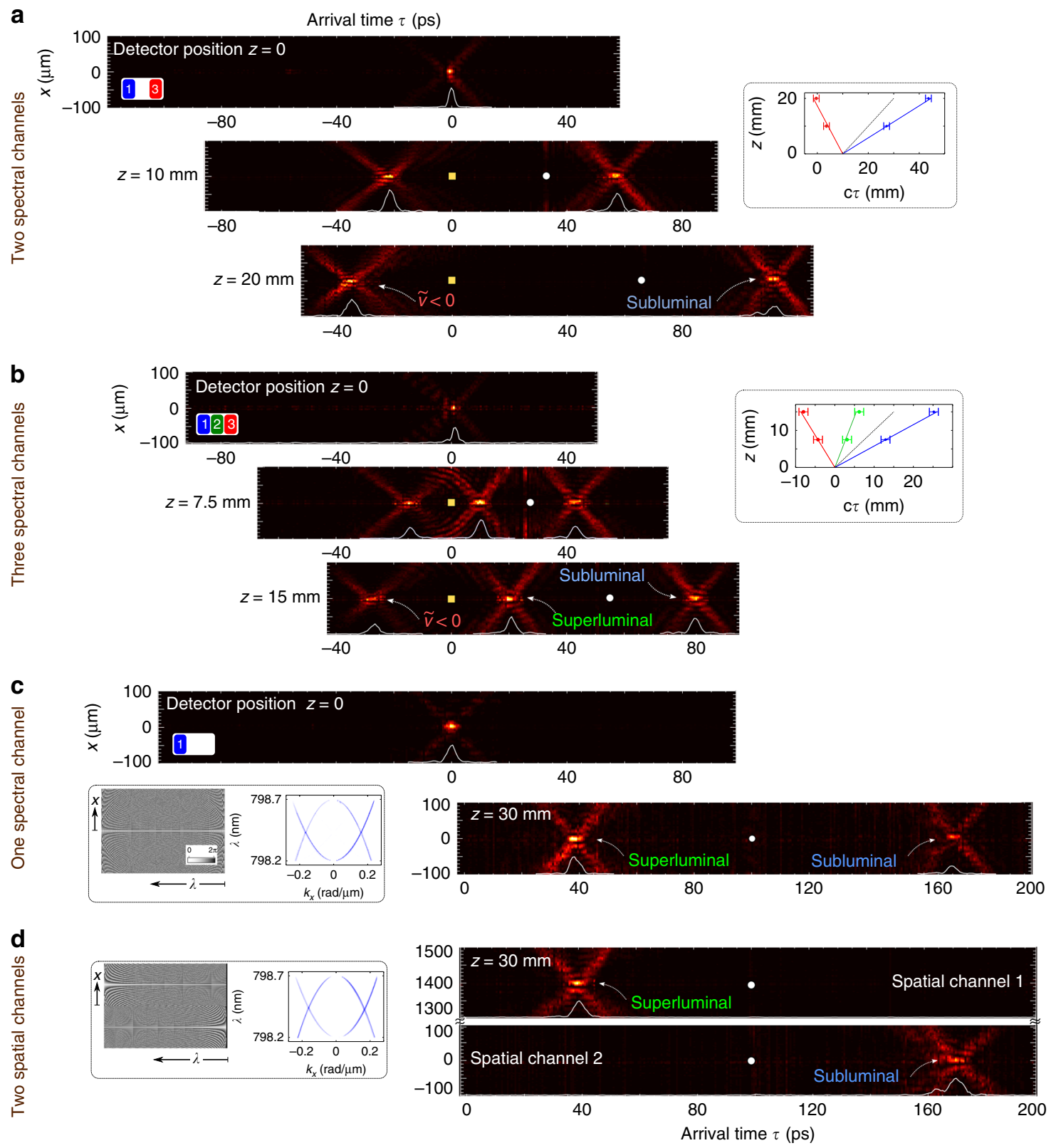

Fig. 4 Propagation of ST wave packets in different or the same spectral or spatial channels. a The panels record the arrival time of subluminal (Ch1) and negative- $\widetilde{v}(\mathrm{Ch} 3)$ wave packets. The two wave packets arrive simultaneously with the detector placed at $z=0$. At $z=20 \mathrm{~mm}$, the subluminal wave packet arrives at $\tau \approx 113$ ps while the negative- $\widetilde{v}$ wave packet arrives at $\tau \approx-35 \mathrm{ps}$ (earlier than the arrival time at the initial detector location of $z=0$ ). The white circle identifies the arrival time of a luminal pulse, whereas the yellow square identifies the $\tau=0$ position. Inset shows the dynamics of the wave-packet centers (similarly to Fig. 3b). b Same as a but with $\mathrm{Ch} 1, \mathrm{Ch} 2$, and $\mathrm{Ch} 3$ all activated. c Two wave packets (subluminal with $\theta_{1}=30^{\circ}$ and superluminal with $\theta_{2}$ $=70^{\circ}$ ) occupy the same spectral channel initially overlap when the detector is placed at $z=0$, and subsequently separate at $z=30 \mathrm{~mm}$. Inset shows the SLM 2D phase distribution required (interleaved distributions from Ch1 and Ch2 in Fig. 2b) and the measured overlapping spatio-temporal spectra. $\mathbf{d}$ Same as $\mathbf{c}$ but with the wave packets in two spatial channels displaced along $x$.

distribution imparted to Ch1 is extended across all three spectral channels to a $\sim 1.5-\mathrm{nm}$ bandwidth.

The measurement results are shown in Fig. 5. First, we synthesize three ST wave packets of on-axis temporal widths of $T_{1} \approx 4 \mathrm{ps}, T_{2} \approx 2.2 \mathrm{ps}$, and $T_{3} \approx 1.3 \mathrm{ps}$, corresponding to bandwidths of $0.5 \mathrm{~nm}(\mathrm{Ch} 1), 1 \mathrm{~nm}(\mathrm{Ch} 1+\mathrm{Ch} 2)$, and $1.5 \mathrm{~nm}(\mathrm{Ch} 1+$ $\mathrm{Ch} 2+\mathrm{Ch} 3)$, respectively. The insets in Fig. 5 show the spatiotemporal profiles of the three wave packets, all having the same group velocity $\widetilde{v}=0.58 c\left(\theta=30^{\circ}\right)$. Next we trace the on-axis intensity for two positions of the detectors: an initial position of $z$ $=0$ and then at $z=20 \mathrm{~mm}$. The group delay experienced by the three ST wave packets $\sim 115$ ps is independent of the pulse width, resulting in a linear increase in DBP with the bandwidth (inversely with the pulse width). Here, the values of the DBP are $\approx 29,52$, and 89 , respectively.

Finally, we examine the limit on miniaturizing the physical length of the delay line. This involves two independent aspects: reduction of the propagation distance needed to realize the relative group delays, and reduction of the size of the experimental apparatus that encodes the ST wave packet structure. With respect to the propagation distance, increasing the deviation of the group velocity $\widetilde{v}=c \tan \theta$ from $c$ (i.e., the deviation of $\theta$ from $45^{\circ}$ ), a large differential group delay can be realized over a short distance. We plot in Fig. 6 the measured 


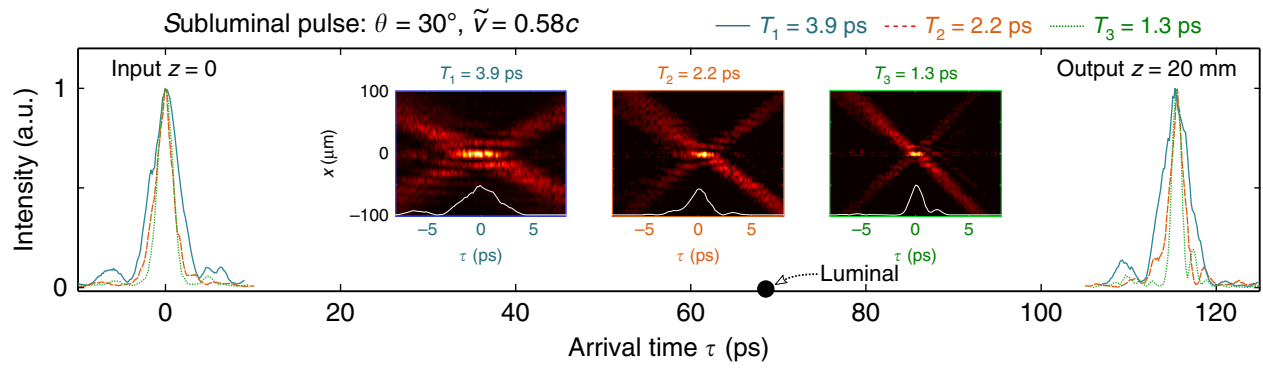

Fig. 5 Increase in the DBP with reduced pulse width (increased temporal spectral bandwidth). We plot the on-axis intensity profile $I(0, z, \tau)$ of three $S T$ wave packets of different widths $\left(T_{1} \approx 3.9 \mathrm{ps}, T_{2} \approx 2.2 \mathrm{ps}\right.$, and $\left.T_{3} \approx 1.3 \mathrm{ps}\right)$ but identical group velocity $(\widetilde{v}=0.58 \mathrm{c})$ recorded at two detector positions: $z=0$ (left) and $z=20 \mathrm{~mm}$ (right). The pulses undergo the same delay of $\sim 115 \mathrm{ps}$ with minimal pulse reshaping. Insets are the spatio-temporal profiles of the wave packets.

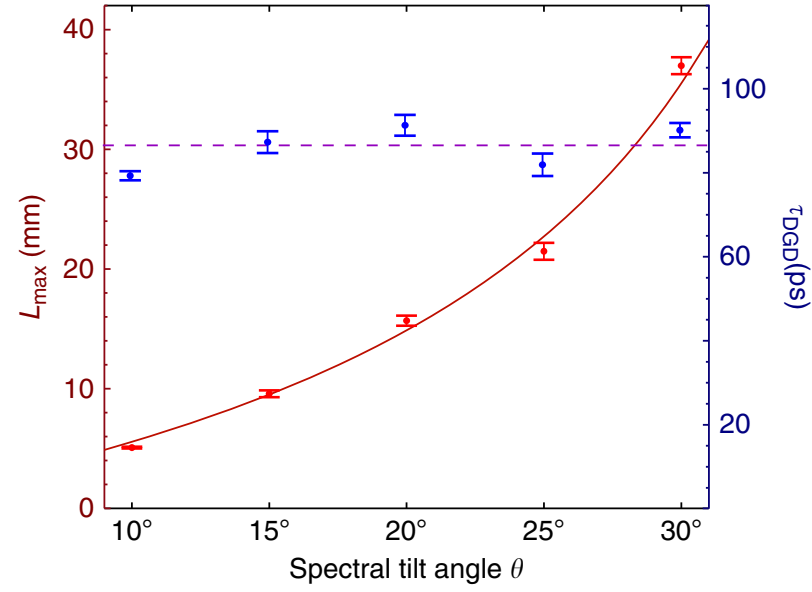

Fig. 6 Propagation distance $L_{\max }$ and differential group delay $\tau_{\mathrm{DGD}}$ against the spectral tilt angle $\theta ; \tau_{\mathrm{DGD}}$ is the difference in group delay between the ST wave packet traveling at a group velocity $\widetilde{\mathbf{v}}=\boldsymbol{c} \tan \theta$ and a luminal reference pulse traveling at $\widetilde{\boldsymbol{v}}=\boldsymbol{c}$. The propagation distance is extracted from the on-axis time-averaged intensity profiles $I(x, z)$, where $I\left(0, L_{\max }\right)=\frac{1}{e} l(0,0)$. The error bars correspond to $\delta L=\Delta z / \sqrt{2}$, where $\Delta z$ is the measurement step along $z$.

propagation distance $L_{\max }$ and the associated differential group delay for subluminal pulses with respect to a luminal pulse. The measurements made use of ST wave packets with spectral tilt angles in the range $10^{\circ}<\theta<30^{\circ}$, corresponding to group velocities in the range $0.017 c<\widetilde{v}<0.58 c$. Note that the differential group delay remains fixed at $\sim 85$ ps independently of $\theta, \widetilde{v}$, and the pulse width $T$, and is instead determined by the spectral uncertainty ${ }^{59}$. At the lower limit of $\theta=10^{\circ}$, the differential group delay is achieved over a distance of only $5 \mathrm{~mm}$. This demonstrates the possibility of constructing compact optical delay lines with large DBP. With respect to the size of the experimental apparatus that encodes the spatio-temporal spectral structure necessary to realize the target group velocity, more research is needed. Our proof-ofconcept realization made use of a system constructed of standard optical components selected for convenience. It is an open question whether a metasurface ${ }^{81}$, or perhaps a pair of metasurfaces separated by free-space propagation for some distance, can introduce the requisite spatio-temporal spectral structure into the optical field. This would lead to a dramatic reduction in the apparatus volume. Moreover, we have recently demonstrated the coupling of ST wave packets, similar to those utilized here, into planar waveguides ${ }^{82}$. This bodes well for potential on-chip realizations of the inline delay line reported here.

\section{Discussion}

We have shown here that ST wave packets can provide a verstaile platform for large controllable inline differential group delays in free space by assigning different group velocities through spatiotemporal spectral-phase modulation. In this regard, ST wave packets offer the advantage of symmetrizing the treatment of the subluminal and superluminal regimes, which are equally useful in an optical buffer, in contrast to the divergence between the physical realizations of slow-light and fast-light (with the latter usually being significantly more challenging). The ST wave packets undergo minimal spreading in space and time, and hence minimal attenuation, while undergoing in one example a differential group delay of $\sim 160$ ps between a pair of ST wave packets over a distance of $40 \mathrm{~mm}$. To put this value in perspective, we note that spatial structuring in refs. ${ }^{23,24}$ resulted in differential group delays of $\sim 10$ 's of femtosecond over a distance of $1 \mathrm{~m}$, so that our result presents an improvement of $\sim 6$ orders-of-magnitude; whereas in refs. ${ }^{54-56}$, X-waves revealed differential group delays of $\sim 100$ 's of femtoseconds over $\sim 10 \mathrm{~cm}$, so that our result corresponds to a boost of $\sim 4$ orders-of-magnitude.

Note that the group velocity does not necessarily coincide with the information velocity ${ }^{83}$. The case of a pulse propagating in a resonant medium displaying strong anomalous dispersion in which the group velocity can be superluminal or even negative was studied more than a century ago by Sommerfeld and Brillouin ${ }^{84}$ (and more recently in the context of fast-light system $^{8}$ ) where it was shown that the information velocity does not exceed $c^{85}$. Recently, Saari has studied in detail superluminal X-waves, which represent a class of ST wave packets, from the perspective of Poynting's vector and field energy distribution thereby confirming that the energy velocity does not exceed $c$ despite the superluminal $\widetilde{v}^{68,69}$. Indeed, $\widetilde{v}$ is the speed of the peak of the wave packet, whereas the information is causally related to non-analytic points in the pulse that result from turning a signal on and off ${ }^{85}$. In our case, despite propagating in free space rather than in a dispersive medium, ST wave packets having superluminal and negative- $\widetilde{v}$ do not violate relativisitic causality. As we showed in ref. ${ }^{59}$, any ST wave packet traveling at a group velocity $\widetilde{v}$ is accompanied by a pilot envelope traveling at a group velocity $c$, which also introduces a latency equal to the width of the pilot envelope (the inverse of the spectral uncertainty) into the path of the ST wave packets. The superluminal ST wave packet advances with respect to the pilot envelope, but when the ST wave packet reaches its edge, rapid deformation sets in. As a result, relativistic causality is not violated. We envision using the peak of the ST wave packet to mark the arrival of each information bit, and the relative group delays due to the different group velocities are therefore realized between bits from distinct data streams, while the whole 
stream is initially delayed by the latency period equal to the width of the pilot envelope.

Such an optical delay line is a critical component of an optical buffer. Several crucial steps remain before achieving this goal, including extending this experimental realization to the telecommunications band at $\sim 1.5 \mu \mathrm{m}$, making use of data streams to test the possibility of alleviating data packet contention at a switch, in addition to evaluating the required resources for our system (especially in terms of overall physical footprint) in comparison to alternative approaches. Furthermore, extending the transverse confinement of ST wave packets to both transverse directions (an optical needle ${ }^{47,48}$ ) rather than the single dimension (light sheet) demonstrated here will enable their coupling to optical waveguides, fibers, and other photonic devices.

It is useful to consider the potential for scaling our strategy to the requirements of a telecommunications network. The freespace configuration used in our experiments is not far removed from commercially available wavelength-selective switches and dynamical gain-equalizers. Such devices typically disperse the spectrum of the optical signal spatially followed by the use of a SLM to introduce functionalities such as channel blocking, dispersion compensation, and channel equalization. Indeed, these devices serve as the main components in reconfigurable optical add-drop multiplexers and can provide spectral dispersers with $<10 \mathrm{GHz}$ resolution in the telecommunication $\mathrm{C}$ band. Given that most wavelength-division multiplexing (WDM) networks operate with signal channels on a $200 \mathrm{GHz}$ grid, the $10 \mathrm{GHz}$ resolution would enable 20 SLM pixels per wavelength band on a typical SLM, which may be sufficient to induce the desired delays using our ST wave packet approach. Therefore, our strategy is in principle compatible with current telecommunications systems.

The unique characteristics of ST wave packets demonstrated here pave the way to such exotic possibilities as colliding copropagating or even counter-propagating optical pulses produced by the same source. Such scenarios could be useful in a variety of applications, including laser machining, laser fusion, and electron acceleration ${ }^{86}$. Finally, we note that another promising approach for the spatio-temporal synthesis of optical wave packets of controllable group velocity has also been recently proposed and investigated ${ }^{87-89}$.

\section{Methods}

Theory for ST wave packets. The spatio-temporal spectrum of a ST wave packet occupies a reduced-dimensionality spectral subspace with respect to traditional wave packets ${ }^{42}$. We consider here one transverse dimension $x$, and the free-space dispersion relationship $k_{x}^{2}+k_{z}^{2}=\left(\frac{\omega}{c}\right)^{2}$ is geometrically represented by the surface of the cone; here $z$ is the axial coordinate along the propagation direction, $\omega$ is the temporal frequency, and $c$ is the speed of light in vacuum. The spatio-temporal spectrum of a typical pulsed beam occupies a $2 \mathrm{D}$ patch on the surface of the lightcone corresponding to a finite spatial bandwidth (that determines the width of the beam profile) and a finite temporal bandwidth (that determines the pulse linewidth). A ST wave packet also has finite spatial and temporal bandwidths, but instead of being represented by a $2 \mathrm{D}$ patch on the light-cone, it lies along the intersection of the light-cone with a tilted spectral plane described by the equation:

$$
\Omega=\left(k_{z}-k_{\mathrm{o}}\right) c \tan \theta,
$$

where $\Omega=\omega-\omega_{\mathrm{o}}, \omega_{\mathrm{o}}$ is a fixed frequency, and $k_{\mathrm{o}}=\omega_{\mathrm{o}} / c$ is the corresponding freespace wave number; see Fig. 1a, right insets. As a result, each spatial frequency $k_{x}$ is associated with a single temporal frequency $\omega$, with a delta-function correlation whose functional form depends on $\theta, k_{x}=k_{x}(\omega, \theta)^{42,70}$. Consequently, the envelope $\psi(x, z, t)$ of the wave packet $E(x, z, t)=e^{i\left(k_{\mathrm{o}} z-\omega_{\mathrm{o}} t\right)} \psi(x, z, t)$ becomes

$$
\psi(x, z, t)=\int d \Omega \widetilde{\psi}(\Omega) e^{i k_{x}(\Omega, \theta) x} e^{-i \Omega(t-z \widetilde{v v}}=\psi(x, 0, t-z / \widetilde{v}),
$$

which is a propagation-invariant wave packet traveling at a group velocity of $\widetilde{v}=c \tan \theta, \widetilde{\psi}(\Omega)$ is the Fourier transform of $\psi(0,0, t)$, and $k_{x}(\omega, \theta)$ is the equation of the conic section at the intersection of the light-cone with the spectral plane after being projected onto the $\left(k_{x}, \frac{\omega}{c}\right)$-plane $e^{70}$; see Figs. 1a and $2 \mathrm{c}$. The type of conic section involved depends on the value of $\theta$ : when $\theta=0$ it is a circle; when $0<\theta<$ $45^{\circ}$ or $135^{\circ}<\theta<180^{\circ}$ an ellipse; when $45^{\circ}<\theta<135^{\circ}$ a hyperbola; when $\theta=45^{\circ}$ a straight line; and when $\theta=135^{\circ}$ a parabola ${ }^{70}$. In all cases, the projection of the spatio-temporal spectrum onto the $\left(k_{z}, \frac{\omega}{c}\right)$-plane is a straight line making an angle $\theta$ with the $k_{z}$-axis; see Fig. 2 d.

In our experiments we divide the available spectrum into three segments. Therefore, the synthesized ST wave packets are in general the sum of three individual ST wave packets having the form,

$$
\begin{aligned}
\psi(x, z, t) & =\psi_{1}(x, z, t)+\psi_{2}(x, z, t)+\psi_{3}(x, z, t) \\
& =\psi_{1}\left(x, 0, t-z / \widetilde{v}_{1}\right)+\psi_{2}\left(x, 0, t-z / \widetilde{v}_{2}\right)+\psi_{3}\left(x, 0, t-z / \widetilde{v}_{3}\right) .
\end{aligned}
$$

Here $\widetilde{v}_{j}=c \tan \theta_{j}, j=1,2,3$, is the group velocity of each wave packet, and $\theta_{j}$ is the spectral tilt angle of the spectral plane associated with each wave packet. The envelope of each wave packet is given by

$$
\psi_{j}(x, z, t)=\int d \Omega \widetilde{\psi}_{j}(\Omega) e^{i k_{x}\left(\Omega, \theta_{j}\right) x} e^{-i \Omega(t-z \widetilde{v})}=\psi_{j}\left(x, 0, t-z / \widetilde{v}_{j}\right),
$$

where $\widetilde{\psi}_{j}(\Omega)$ is the Fourier transform of $\psi_{j}(0,0, t)$, and each spectral channel determines the domain of integration for $\omega$. Each channel has a distinct value of $\omega_{\mathrm{o}}$ and $k_{\mathrm{o}}$. In Fig. $2 \mathrm{~d}$ we shift the three values of $k_{\mathrm{o}, j}$ to coincide with each other for clarity.

In our experiments, we have $\theta_{1}=30^{\circ}$ (subluminal, conic section is an ellipse); $\theta_{2}=70^{\circ}$ (superluminal, hyperbola); and $\theta_{3}=120^{\circ}$ (negative- $\widetilde{v}$, hyperbola). The spectrum that is spatially resolved by the diffraction grating and incident on the SLM has a bandwidth of $\sim 1.5 \mathrm{~nm}$. The SLM active area is divided into three sections, each corresponding to $\sim 0.5 \mathrm{~nm}$ of spectrum (Fig. 2a). Within each one of these sections, a $2 \mathrm{D}$ phase pattern $\Phi_{j}$ is implemented (Fig. 2b) that produces the targeted spatio-temporal spectral association $k_{x}^{(j)}\left(\Omega, \theta_{j}\right)$ (Fig. 2c).

\section{Data availability}

The data that support the plots within this paper and other findings of this study are available from the corresponding author upon reasonable request.

Received: 12 October 2019; Accepted: 11 October 2020; Published online: 13 November 2020

\section{References}

1. Richardson, D. J. Filling the light pipe. Science 330, 327-328 (2010).

2. Bozinovic, N. et al. Terabit-scale orbital angular momentum mode division multiplexing in fibers. Science 340, 1545-1548 (2013)

3. Li, G., Bai, N., Zhao, N. \& Xia, C. Space-division multiplexing: the next frontier in optical communication. Adv. Opt. Photon. 6, 413-487 (2014)

4. Zhao, N., Li, X., Li, G. \& Kahn, J. M. Capacity limits of spatially multiplexed free-space communication. Nat. Photon. 9, 822-826 (2015).

5. Wang, C. et al. Integrated lithium niobate electro-optic modulators operating at CMOS-compatible voltages. Nature 562, 101-104 (2018).

6. Boyd, R. W., Gauthier, D. J. \& Gaeta, A. L. Applications of slow light in telecommunications. Opt. Photon. N. 17, 18-23 (2006).

7. Krauss, T. F. Why do we need slow light? Nat. Photon. 2, 448-450 (2008)

8. Boyd, R. W. \& Gauthier, D. J. Controlling the velocity of light pulses. Science 326, 1074-1077 (2009).

9. Hau, L. V., Harris, S. E., Dutton, Z. \& Behroozi, C. Light speed reduction to 17 $\mathrm{m}$ per second in an ultracold atomic gas. Nature 397, 594-598 (1999).

10. Kash, M. M. et al. Ultraslow group velocity and enhanced nonlinear optical effects in a coherently driven hot atomic gas. Phys. Rev. Lett. 82, 5229-5232 (1999).

11. Song, K. Y., Herráez, M. G. \& Thévenaz, L. Gain-assisted pulse advancement using single and double Brillouin gain peaks in optical fibers. Opt. Express 13, 9758-9765 (2005)

12. Okawachi, Y. et al. Tunable all-optical delays via Brillouin slow light in an optical fiber. Phys. Rev. Lett. 94, 153902 (2005).

13. Vlasov, Y. A., O Boyle, M., Hamann, H. F. \& McNab, S. J. Active control of slow light on a chip with photonic crystal waveguides. Nature 438, 65-69 (2005).

14. Krauss, T. F. Slow light in photonic crystal waveguides. J. Phys. D. 40, 2666-2670 (2007)

15. Baba, T. Slow light in photonic crystals. Nat. Photon. 2, 465-473 (2008).

16. Khurgin, J. B. \& Tucker, R. S. (eds.) Slow Light: Science and Applications (CRC Press, Boca Raton, 2008).

17. Tucker, R. S., Ku, P. C. \& Chang-Hasnain, C. J. Delay-bandwidth product and storage density in slow-light optical buffers. Electron. Lett. 41, 208-209 (2005).

18. Tucker, R. S., Ku, P. C. \& Chang-Hasnain, C. J. Slow-light optical buffers: capabilities and fundamental limitations. J. Lightwave Technol. 23, 4046-4066 (2005).

19. Parra, E. \& Lowell, J. R. Toward applications of slow light technology. Opt. Photon. N. 18, 40-45 (2007). 
20. Yanik, M. F. \& Fan, S. Stopping light all optically. Phys. Rev. Lett. 92, 083901 (2004).

21. Xu, Q., Dong, P. \& Lipson, M. Breaking the delay-bandwidth limit in a photonic structure. Nat. Phys. 3, 406-410 (2007).

22. Chang, Y.-C., Roberts, S. P., Stern, B. \& Lipson, M. Resonance-free light recycling. Preprint at arXiv: 1710.02891 (2017).

23. Giovannini, D. et al. Spatially structured photons that travel in free space slower than the speed of light. Science 347, 857-860 (2015).

24. Bouchard, F., Harris, J., Mand, H., Boyd, R. W. \& Karimi, E. Observation of subluminal twisted light in vacuum. Optica 3, 351-354 (2016).

25. Zapata-Rodríguez, C. J., Porras, M. A. \& Miret, J. J. Free-space delay lines and resonances with ultraslow pulsed Bessel beams. J. Opt. Soc. Am. A 25, 2758-2763 (2008).

26. Alfano, R. R. \& Nolan, D. A. Slowing of Bessel light beam group velocity. Opt. Commun. 361, 25-27 (2016).

27. Saari, P. Comments on "Slowing of Bessel light beam group velocity". Opt. Commun. 392, 300-301 (2017).

28. Tsakmakidis, K. L. et al. Breaking Lorentz reciprocity to overcome the timebandwidth limit in physics and engineering. Science 356, 1260-1264 (2017).

29. Tsang, M. Quantum limits on the time-bandwidth product of an optical resonator. Opt. Lett. 43, 150-153 (2018).

30. Mann, S. A., Sounas, D. L. \& Alú, A. Nonreciprocal cavities and the timebandwidth limit. Optica 6, 104-110 (2019).

31. Brittingham, J. N. Focus wave modes in homogeneous Maxwell's equations: transverse electric mode. J. Appl. Phys. 54, 1179-1189 (1983).

32. Ziolkowski, R. W. Exact solutions of the wave equation with complex source locations. J. Math. Phys. 26, 861-863 (1985).

33. Lu, J.-Y. \& Greenleaf, J. F. Nondiffracting X waves - exact solutions to freespace scalar wave equation and their finite aperture realizations. IEEE Trans. Ultrason. Ferroelec. Freq. Control 39, 19-31 (1992).

34. Saari, P. \& Reivelt, K. Evidence of X-shaped propagation-invariant localized light waves. Phys. Rev. Lett. 79, 4135-4138 (1997).

35. Reivelt, K. \& Saari, P. Localized wave solutions of the scalar homogeneous wave equation and their optical implementation. Preprint at arxiv: physics/ 0309079 (2003).

36. Kiselev, A. P. Localized light waves: paraxial and exact solutions of the wave equation (a review). Opt. Spectrosc. 102, 603-622 (2007).

37. Turunen, J. \& Friberg, A. T. Propagation-invariant optical fields. Prog. Opt. 54, $1-88$ (2010).

38. Hernández-Figueroa, H. E., Recami, E. \& Zamboni-Rached, M. (eds) Nondiffracting Waves (Wiley-VCH, 2014).

39. Donnelly, R. \& Ziolkowski, R. Designing localized waves. Proc. R. Soc. Lond. A 440, 541-565 (1993).

40. Porras, M. A. Gaussian beams diffracting in time. Opt. Lett. 42, 4679-4682 (2017).

41. Efremidis, N. K. Spatiotemporal diffraction-free pulsed beams in free-space of the Airy and Bessel type. Opt. Lett. 42, 5038-5041 (2017).

42. Kondakci, H. E. \& Abouraddy, A. F. Diffraction-free space-time beams. Nat. Photon. 11, 733-740 (2017).

43. Porras, M. A. Nature, diffraction-free propagation via space-time correlations, and nonlinear generation of time-diffracting light beams. Phys. Rev. A 97, 063803 (2018).

44. Longhi, S. Gaussian pulsed beams with arbitrary speed. Opt. Express 12, 935-940 (2004).

45. Saari, P. \& Reivelt, K. Generation and classification of localized waves by Lorentz transformations in Fourier space. Phys. Rev. E 69, 036612 (2004)

46. Kondakci, H. E. \& Abouraddy, A. F. Diffraction-free pulsed optical beams via space-time correlations. Opt. Express 24, 28659-28668 (2016).

47. Parker, K. J. \& Alonso, M. A. The longitudinal iso-phase condition and needle pulses. Opt. Express 24, 28669-28677 (2016).

48. Wong, L. J. \& Kaminer, I. Abruptly focusing and defocusing needles of light and closed-form electromagnetic wavepackets. ACS Photon. 4, 1131-1137 (2017).

49. Wong, L. J. \& Kaminer, I. Ultrashort tilted-pulsefront pulses and nonparaxial tilted-phase-front beams. ACS Photon. 4, 2257-2264 (2017).

50. Salo, J. \& Salomaa, M. M. Diffraction-free pulses at arbitrary speeds. J. Opt. A 3, 366-373 (2001).

51. Zapata-Rodríguez, C. J. \& Porras, M. A. X-wave bullets with negative group velocity in vacuum. Opt. Lett. 31, 3532-3534 (2006).

52. Valtna, H., Reivelt, K. \& Saari, P. Methods for generating wideband localized waves of superluminal group velocity. Opt. Commun. 278, 1-7 (2007).

53. Zamboni-Rached, M. \& Recami, E. Subluminal wave bullets: exact localized subluminal solutions to the wave equations. Phys. Rev. A 77, 033824 (2008).

54. Bonaretti, F., Faccio, D., Clerici, M., Biegert, J. \& Di Trapani, P. Spatiotemporal amplitude and phase retrieval of Bessel-X pulses using a Hartmann-Shack sensor. Opt. Express 17, 9804-9809 (2009).
55. Bowlan, P. et al. Measuring the spatiotemporal field of ultrashort Bessel-X pulses. Opt. Lett. 34, 2276-2278 (2009).

56. Kuntz, K. B. et al. Spatial and temporal characterization of a bessel beam produced using a conical mirror. Phys. Rev. A 79, 043802 (2009).

57. Yessenov, M., Bhaduri, B., Kondakci, H. E. \& Abouraddy, A. F. Weaving the rainbow: space-time optical wave packets. Opt. Photon. N. 30, 34-41 (2019).

58. Kondakci, H. E. \& Abouraddy, A. F. Optical space-time wave packets of arbitrary group velocity in free space. Nat. Commun. 10, 929 (2019).

59. Yessenov, M. et al. What is the maximum differential group delay achievable by a space-time wave packet in free space? Opt. Express 27, 12443-12457 (2019).

60. Bhaduri, B., Yessenov, M. \& Abouraddy, A. F. Meters-long propagation of diffraction-free space-time light sheets. Opt. Express 26, 20111-20121 (2018).

61. Bhaduri, B. et al. Broadband space-time wave packets propagating for $70 \mathrm{~m}$. Opt. Lett. 44, 2073-2076 (2019).

62. Kondakci, H. E. \& Abouraddy, A. F. Airy wavepackets accelerating in spacetime. Phys. Rev. Lett. 120, 163901 (2018).

63. Kondakci, H. E. \& Abouraddy, A. F. Self-healing of space-time light sheets. Opt. Lett. 43, 3830-3833 (2018).

64. Bhaduri, B., Yessenov, M. \& Abouraddy, A. F. Space-time wave packets that travel in optical materials at the speed of light in vacuum. Optica 6, 139-146 (2019).

65. Kondakci, H. E., Nye, N. S., Christodoulides, D. N. \& Abouraddy, A. F. Tiltedpulse-front space-time wave packets. ACS Photon. 6, 475-481 (2019).

66. Shaarawi, A. M. \& Besieris, I. M. Relativistic causality and superluminal signalling using X-shaped localized waves. J. Phys. A 33, 7255-7263 (2000).

67. Saari, P. Reexamination of group velocities of structured light pulses. Phys. Rev. A 97, 063824 (2018).

68. Saari, P., Rebane, O. \& Besieris, I. Reexamination of energy flow velocities of non-diffracting localized waves. Phys. Rev. A 100, 013849 (2019).

69. Saari, P. \& Besieris, I. Reactive energy in nondiffracting localized waves. Phys Rev. A 101, 023812 (2020).

70. Yessenov, M., Bhaduri, B., Kondakci, H. E. \& Abouraddy, A. F. Classification of propagation-invariant space-time light-sheets in free space: theory and experiments. Phys. Rev. A 99, 023856 (2019).

71. Reivelt, K. \& Saari, P. Experimental demonstration of realizability of optical focus wave modes. Phys. Rev. E 66, 056611 (2002).

72. Faccio, D. et al. Spatio-temporal reshaping and $\mathrm{X}$ wave dynamics in optical filaments. Opt. Express 15, 13077-13095 (2007).

73. Kondakci, H. E. et al. Synthesizing broadband propagation-invariant spacetime wave packets using transmissive phase plates. Opt. Express 26, 13628-13638 (2018)

74. Yessenov, M. et al. Non-diffracting broadband incoherent space-time fields. Optica 6, 598-607 (2019d).

75. Yessenov, M. \& Abouraddy, A. F. Changing the speed of optical coherence in free space. Opt. Lett. 44, 5125-5128 (2019).

76. Weiner, A. M. Femtosecond pulse shaping using spatial light modulators. Rev Sci. Instrum. 71, 1929-1960 (2000).

77. Weiner, A. M. Ultrafast Optics (John Wiley \& Sons, Inc., 2009).

78. Adams, D. E., Planchon, T. A., Hrin, A., Squier, J. A. \& Durfee, C. G. Characterization of coupled nonlinear spatiospectral phase following an ultrafast self-focusing interaction. Opt. Lett. 34, 1294-1296 (2009).

79. Lõhmus, M. et al. Diffraction of ultrashort optical pulses from circularly symmetric binary phase gratings. Opt. Lett. 37, 1238-1240 (2012).

80. Sezginer, A. A general formulation of focus wave modes. J. Appl. Phys. 57, 678-683 (1985)

81. Yu, N. \& Capasso, F. Flat optics with designer metasurfaces. Nat. Mater. 13, 139-150 (2014)

82. Shiri, A., Yessenov, M., Webster, S., Schepler, K. L. \& Abouraddy, A. F. Hybrid guided space-time optical modes in unpatterned films. Preprint at arXiv: 2001.01991 (2020).

83. Smith, R. L. The velocities of light. Am. J. Phys. 38, 978-783 (1970).

84. Brillouin, L. Wave Propagation and Group Velocity (Academic Press, New York, 1960).

85. Stenner, M. D., Gauthier, D. J. \& Neifeld, M. A. The speed of information in a 'fast-light' optical medium. Nature 425, 695-698 (2003).

86. Jolly, S. W. Influence of longitudinal chromatism on vacuum acceleration by intense radially polarized laser beams. Opt. Lett. 44, 1833-1836 (2019).

87. Sainte-Marie, A., Gobert, O. \& Quéré, F. Controlling the velocity of ultrashort light pulses in vacuum through spatio-temporal couplings. Optica 4, 1298-1304 (2017)

88. Froula, D. H. et al. Spatiotemporal control of laser intensity. Nat. Photon. 12, 262-265 (2018).

89. Jolly, S. W., Gobert, O., Jeandet, A. \& Quéré, F. Controlling the velocity of a femtosecond laser pulse using refractive lenses. Opt. Express 28, 4888-4897 (2020). 


\section{Acknowledgements}

We thank Matteo Clerici, Kenneth L. Schepler, and Abbas Shiri for helpful discussions. This work was supported by the U.S. Office of Naval Research (ONR) under contracts N00014-17-1-2458 and N00014-19-1-2192, and ONR MURI contract N00014-20-12789.

\section{Author contributions}

A.F.A. and M.Y. developed the concept and designed the experiment. M.Y. carried out the experiment and analyzed the data. B.B. assisted with the experiment. A.F.A. and P.J. D. supervised the research. All authors contributed to writing the paper.

\section{Competing interests}

The authors declare no competing interests.

\section{Additional information}

Correspondence and requests for materials should be addressed to A.F.A.

Peer review information Nature Communications thanks Liang Jie Wong and the other anonymous reviewer for their contribution to the peer review of this work.
Reprints and permission information is available at http://www.nature.com/reprint

Publisher's note Springer Nature remains neutral with regard to jurisdictional claims in published maps and institutional affiliations.

(c) (i) Open Access This article is licensed under a Creative Commons Attribution 4.0 International License, which permits use, sharing, adaptation, distribution and reproduction in any medium or format, as long as you give appropriate credit to the original author(s) and the source, provide a link to the Creative Commons license, and indicate if changes were made. The images or other third party material in this article are included in the article's Creative Commons license, unless indicated otherwise in a credit line to the material. If material is not included in the article's Creative Commons license and your intended use is not permitted by statutory regulation or exceeds the permitted use, you will need to obtain permission directly from the copyright holder. To view a copy of this license, visit http://creativecommons.org/ licenses/by/4.0/.

(C) The Author(s) 2020 